\title{
Nanoengineered drug-releasing Ti wires as an alternative for local delivery of chemotherapeutics in the brain
}

This article was published in the following Dove Press journal:

International Journal of Nanomedicine

18 April 2012

Number of times this article has been viewed

\author{
Karan Gulati ${ }^{1,2}$ \\ Moom Sinn Aw' \\ Dusan Losic ${ }^{1,2}$ \\ I Ian Wark Research Institute, \\ The University of South Australia, \\ Adelaide, SA, Australia; ${ }^{2}$ School of \\ Chemical Engineering, The University \\ of Adelaide, Adelaide, SA, Australia
}

Correspondence: Dusan Losic School of Chemical Engineering, The University of Adelaide, NI 19 North Engineering Building, Adelaide, SA 5005, Australia

Tel +6I 883034648

Fax +6I 883034373

Email dusan.losic@adelaide.edu.au
Abstract: The blood-brain barrier (BBB) blocks the passage of active molecules from the blood which makes drug delivery to the brain a challenging problem. Oral drug delivery using chemically modified drugs to enhance their transport properties or remove the blocking of drug transport across the BBB is explored as a common approach to address these problems, but with limited success. Local delivery of drugs directly to the brain interstitium using implants such as polymeric wafers, gels, and catheters has been recognized as a promising alternative particularly for the treatment of brain cancer (glioma) and neurodegenerative disorders. The aim of this study was to introduce a new solution by engineering a drug-releasing implant for local drug delivery in the brain, based on titanium (Ti) wires with titania nanotube (TNT) arrays on their surfaces. Drug loading and drug release characteristics of this system were explored using two drugs commonly used in oral brain therapy: dopamine (DOPA), a neurotransmitter agent; and doxorubicin (DOXO), an anticancer drug. Results showed that TNT/Ti wires could provide a considerable amount of drugs $(>170 \mu \mathrm{g}$ to $1000 \mu \mathrm{g}$ ) with desirable release kinetics and controllable release time (1 to several weeks) and proved their feasibility for use as drug-releasing implants for local drug delivery in the brain.

Purpose: In this report, a new drug-releasing platform in the form of nanoengineered Ti wires with TNT arrays is proposed as an alternative for local delivery of chemotherapeutics in the brain to bypass the BBB. To prove this concept, drug loading and release characteristics of two drugs important for brain therapy (the neurotransmitter DOPA and the anticancer drug DOXO) were explored.

Methods: Titania nanotube arrays on the surface of Ti wires (TNT/Ti) were fabricated using a simple anodization process, followed by separate loading of two drugs (DOPA and DOXO) inside the nanotube structures. The loading and in vitro release characteristics of prepared TNT/ Ti implants were examined using thermogravimetric analysis (TGA) UV-Vis spectroscopy.

Results: Scanning electron microscopy studies confirmed that well-ordered, vertically aligned, densely packed nanotube arrays with an average diameter of $170 \mathrm{~nm}$ and length $70 \mu \mathrm{m}$ were formed on the surface of TNT/Ti wires. TGA results showed a total drug loading of $170 \mu \mathrm{g}$ and $1200 \mu \mathrm{g}$ inside the TNTs for DOPA and DOXO respectively. Two-phase drug release behavior was observed including a fast release (burst) for the first 6 hours and a prolonged slow release phase for 8 days, both with acceptable dosage and desirable release kinetics. The physical, structural, loading and release characteristics of prepared TNT/Ti implants showed several advantages in comparison with existing and clinically proved brain implants.

Conclusion: Our results confirmed that TNT/Ti wires can be successfully employed as a suitable platform to release neurotransmitters such as DOPA and anticancer drugs such as DOXO. Hence, they are a feasible alternative as drug-releasing implants for local drug delivery in the brain to combat neurodegenerative disorders or brain tumors.

Keywords: titania nanotubes, brain implants, local drug delivery, dopamine, doxorubicin 


\section{Introduction}

To address the limitations of conventional and systematic drug delivery such as low drug efficacy, poor bioavailability and biodistribution, drug overdose, and high toxicity, a local drug delivery system to deliver drugs to specific sites is recognized as a promising alternative..$^{1,2}$ One of the most challenging problems in the conventional administration of therapeutics is the delivery of drugs to treat diseases associated with the central nervous system (CNS) and the brain.,4 The brain is a specific organ, isolated from general circulation and characterized by the presence of relatively impermeable endothelial cells with tight junctions, enzymes, receptors and active efflux transport mechanisms, known as the blood-brain barrier (BBB), which blocks the passage of active molecules from the blood compartment to the brain. ${ }^{3}$ As a result most therapeutics have difficulties in effectively reaching the targeted sites in the brain, and hence there is an urgent need to develop advanced drug delivery platforms that are able to bypass this barrier. One approach is to chemically modify drugs or drug vectors to enhance their transport properties, or use co-drug systems for the inhibition of efflux transporters to remove the blocking of drug transport across the BBB. ${ }^{5}$ Several drug delivery systems were developed to bypass the BBB specifically for the treatment of neurodegenerative diseases (Alzheimer's disease and Parkinson's disease) and brain cancer, but with limited success. ${ }^{6-8}$ Also majority of drugs do not have the desirable physicochemical characteristics, such as high lipid solubility, low molecular size, hydrophilic compounds, and/or positive charges, which are necessary to allow passage across the BBB. ${ }^{5}$ These obstacles have resulted in an emphasis on developing alternative delivery modalities based on local delivery of drugs directly to the brain interstitium, particularly focused on treating brain cancer (glioma) and neurodegenerative disorders. ${ }^{9}$

Local drug delivery systems like cerebral infusion methods, polymeric nanoparticles, polymeric wafers or gels, catheters, and pumps were extensively explored in recent years as promising alternatives for the delivery of chemotherapeutic agents into the brain. ${ }^{10-16}$ The development of biodegradable polymer implants (Gliadel R Wafer, MGI Pharma, Bloomington, MN) has been approved by the US Food and Drug Administration and clinically applied for delivering an anticancer drug (carmustine) to the brain. This is the only interstitial chemotherapy device currently available for the treatment of brain tumors. ${ }^{17,18}$ The carmustine wafer ( $14.5 \mathrm{~mm}$ in diameter and $1 \mathrm{~mm}$ thick) containing $7.7 \mathrm{mg}$ of drug in biodegradable polymer (poly[bis(p-carboxyphenoxy)propane-sebacic acid]) is placed in the surgical cavity around the identified tumor and drug is released as a result of polymer degradation for several weeks. ${ }^{19}$ Although the preclinical studies have shown modest efficacy, they serve as adequate proof of principle to demonstrate that drug-releasing implants can safely serve as a medium to bypass the BBB. Consequently, this strategy opens up new opportunities for the development of more advanced implants based on this concept using different nanoengineered platforms, and different drugs for the treatment of a broad range of brain diseases.

In this work we propose a new solution to engineer a drug-releasing implant for local drug delivery in the brain, based on titanium (Ti) wires with titania nanotube (TNT) arrays on the surface. TNT arrays, formed by a selfordering electrochemical anodization process, have been widely recognized as a superior platform for local drug delivery applications particularly in the bones, ${ }^{20-23}$ due to their excellent biocompatibility, the high surface-to-volume ratio, controllable dimensions, tailorable surface chemistry, mechanical/chemical stability and proven capability to load and release a large amount of drugs. In our previous work we demonstrated several strategies for extended release of poorly water-soluble drugs with zero-ordered drug release kinetics from TNTs using plasma polymer/biopolymer coating and polymer micelles as drug nanocarriers. ${ }^{24-28}$ These approaches have demonstrated many advantages for local therapies where sustained and extended delivery of drugs is required. Here, we propose the application of nanoengineered Ti wires with TNT structures on the surface as drug-releasing implants for local delivery of chemotherapeutics in the brain. The proposed system is schematically presented in Figure 1. The TNT/Ti wires are sugested as a brain implant because this design provides simplified surgical implementation inside the brain. To explore the feasibility of this system as a drug carrier for local therapy in the brain, the drug loading and release characteristics of two model drugs relevant to treatment of CNS diseases were investigated. The first, dopamine (dopamine hydrochloride, DOPA) used as a neurotransmitter agent for Parkinson's and Alzheimer disease and, the second doxorubicin (DOXO), a common anticancer drugs for the treatment of brain tumors. Both these drugs are currently applied intravenously, and their therapeutic action is limited as they cannot efficiently cross the BBB to reach the site of action inside the brain.

\section{Materials and methods Materials}

High purity (99.7\%) titanium (Ti) wires (thickness $0.75 \mathrm{~mm}$ ) supplied by Alfa Aesar (Ward Hill, MA) were used as the 


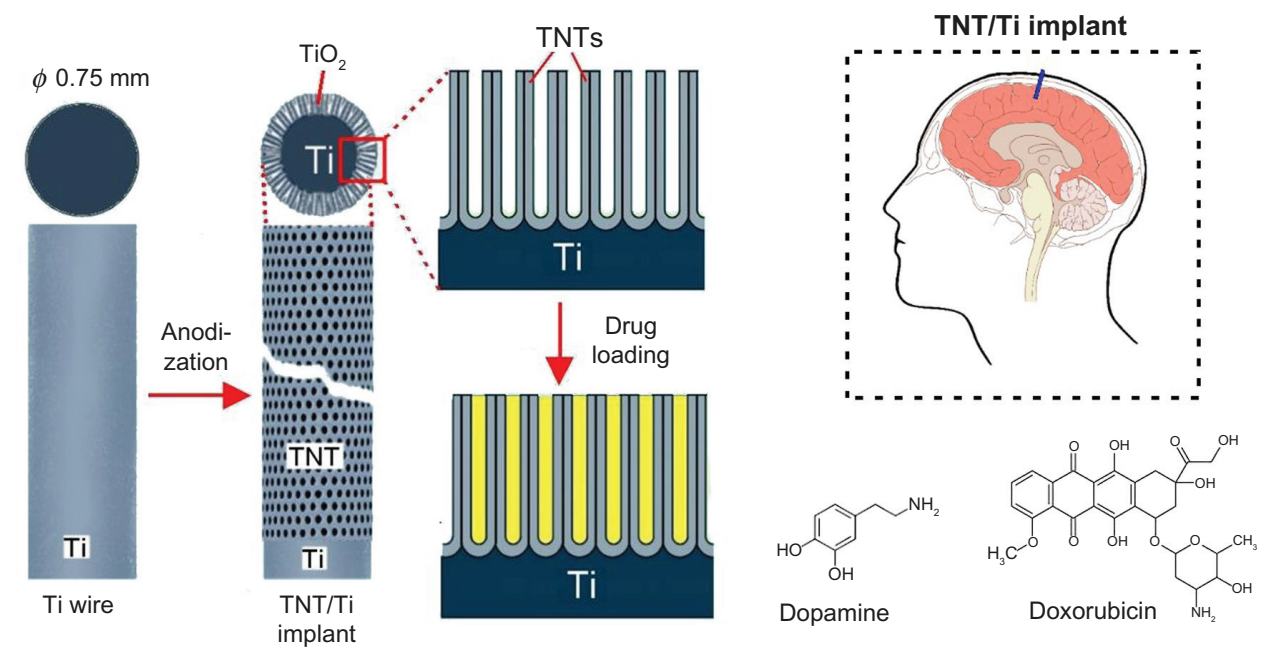

Figure I Scheme of drug-releasing implants for local delivery of therapeutics in the brain based on Ti wire with titania nanotube (TNT) arrays on the surface generated by electrochemical anodization. Dopamine (brain neurotransmitter) and doxorubicin (anticancer drug) were used as model drugs.

Abbreviations: $\mathrm{Ti}$, titanium; $\mathrm{TiO}_{2}$, titanium dioxide; $\mathrm{TNT}$, titania nanotube.

substrate materials. Ethylene glycols, ammonium fluoride $\left(\mathrm{NH}_{4} \mathrm{~F}\right)$, were obtained from Sigma-Aldrich (Sydney, Australia). Dopamine hydrochloride and doxorubicin were purchased from Sigma-Aldrich and InterPharma Pty Ltd (Sydney, Australia) respectively. High purity Milli-Q water, ultra-pure grade (18.2 M $\Omega$ ) sieved through a $0.22 \mu \mathrm{m}$ filter was used for preparation all water solutions throughout this study.

\section{Preparation of titania nanotube arrays on Ti wires (TNT/Ti)}

Titanium wires were cut into equal length of 30-40 mm each, mechanically polished and cleaned by sonication in acetone for 30 minutes prior to anodization. Two anodization steps were performed using a specially designed electrochemical cell and computer-controlled power supply (Agilent, Santa Clara, CA), using previously described procedures. ${ }^{29,30}$ In the first anodization step, a constant voltage of $100 \mathrm{~V}$ was applied for 1 hour in ammonium fluoride/ethylene glycol electrolyte $\left(3 \%\right.$ water and $\left.0.3 \% \mathrm{NH}_{4} \mathrm{~F}\right)$ at $20^{\circ} \mathrm{C}$. The obtained anodic TNT layer was removed (by sonication in methanol), leaving the nanotextured titanium surface for the second anodization. A second anodization was performed at the same voltage at 1-2 hours to yield an ordered array of TNT structures on Ti wire.

\section{Drug loading into TNT/Ti wire}

Drug solutions of DOPA $(0.5 \% \mathrm{w} / \mathrm{v}$ in water) and DOXO $(0.2 \% \mathrm{w} / \mathrm{v}$ in toluene $)$ were prepared and used for loading into $\mathrm{TNT} / \mathrm{Ti}$ wires. Toluene was selected as a solvent for DOXO due to its low water solubility. TNT/Ti wires cut into smaller pieces ( $25 \mathrm{~mm}$ or $10 \mathrm{~mm}$ of active length with TNTs) were fully immersed in the respective DOPA or DOXO solutions to ensure drugs were entirely loaded inside the nanotubes. The wires were rotated every 2-4 hours during deposition. After 1-3 days of loading, the wires were taken out, and dried in air and vacuum to ensure the removal of solvent from the TNTs. Finally, to remove the excess of loaded drug from the TNT surfaces, samples were gently cleaned with a soft tissue after wetting with small amount of phosphate buffered saline.

\section{Structural characterization of TNT/Ti wires}

Structural characterization of the prepared TNT/Ti wires before and after drug loading and release were performed using a field emission scanning electron microscope (SEM, Philips XL 30, Philips Research, Amsterdam, The Netherlands). The samples were cut into small (approximately $5 \mathrm{~mm}$ ) pieces, mounted on a holder with double-sided conductive tape and coated with a layer of platinum 3-5 nm thick. Images with a range of scan sizes at normal incidence and at a $30^{\circ}$ angle were acquired from the top, the bottom surface and cross-sections.

\section{Drug loading characterization}

To quantify the amount of drug loaded inside the TNT/Ti wires, thermogravimetric analysis (TGA) which measures the mass change (weight loss) in the sample as a function of temperature was performed using a Hi-Res Modulated TGA 2950, (TA Instruments, New Castle, DE). To find the correct decomposition range/peak of both the drugs, TGA 
of the two pure drugs (without solvent) was performed in separate experiments. Later the drug-loaded TNT/Ti wire was mounted on the platinum pan and heated from $20^{\circ} \mathrm{C}$ to $800^{\circ} \mathrm{C}$ in the furnace at a scanning rate of $10^{\circ} \mathrm{C} / \mathrm{min}$ under nitrogen gas flow $(50 \mathrm{~mL} / \mathrm{min})$. Only the weight loss peaks identifying the particular drug were taken into consideration to calculate the loading amount. Finally the drug amount present inside the TNTs was evaluated using the TGA analyzing software (Q Series ${ }^{\mathrm{TM}}$ Thermal Analysis, Universal Analysis 2000, TA Instruments).

\section{In vitro drug release characterization}

The release of DOPA and DOXO loaded TNT/Ti wires was investigated using in vitro studies in $5 \mathrm{~mL}$ phosphate buffer ( $\mathrm{pH}$ 7.2), where the amount of released drug was measured using UV-Vis spectroscopy. Measurements were taken at short intervals during the first 6 hours to monitor the initial burst release, followed by repeated measurements every 24 hours to monitor the release until the entire drug amount was released into the buffer solution. During each measurement, an aliquot of $1 \mathrm{~mL}$ was placed in a quartz cuvette ( $1 \mathrm{~mL}$ working volume, $5 \mathrm{~mm}$ path length) and their absorbance measured using a Cary $1 \mathrm{E}$ spectrophotometer (Varian Inc, Palo Alto, CA) to determine the released amount of drug based on a preconstructed calibration curve using the prominent wavelength of the individual drug, $(250 \mathrm{~nm}$ for DOPA and $253 \mathrm{~nm}$ for DOXO. The TNTs samples loaded with DOPA were kept in the dark during the experiments to prevent oxidation of the light-sensitive drug. The corresponding drug concentrations were then calculated from the absorbance values using the calibration curve. The drug release experiment for each drug was carried out in triplicate.

\section{Results and discussion \\ Structural characterization of TNT/Ti wires}

A series of SEM images are presented in Figure 2 to summarize the morphology of prepared TNT/Ti wires. Figure 2A presents a photo of the whole TNT/Ti wire $(25 \mathrm{~mm})$ and a low resolution image of the Ti wire confirming the radial growth of TNT films and their complete coverage of the
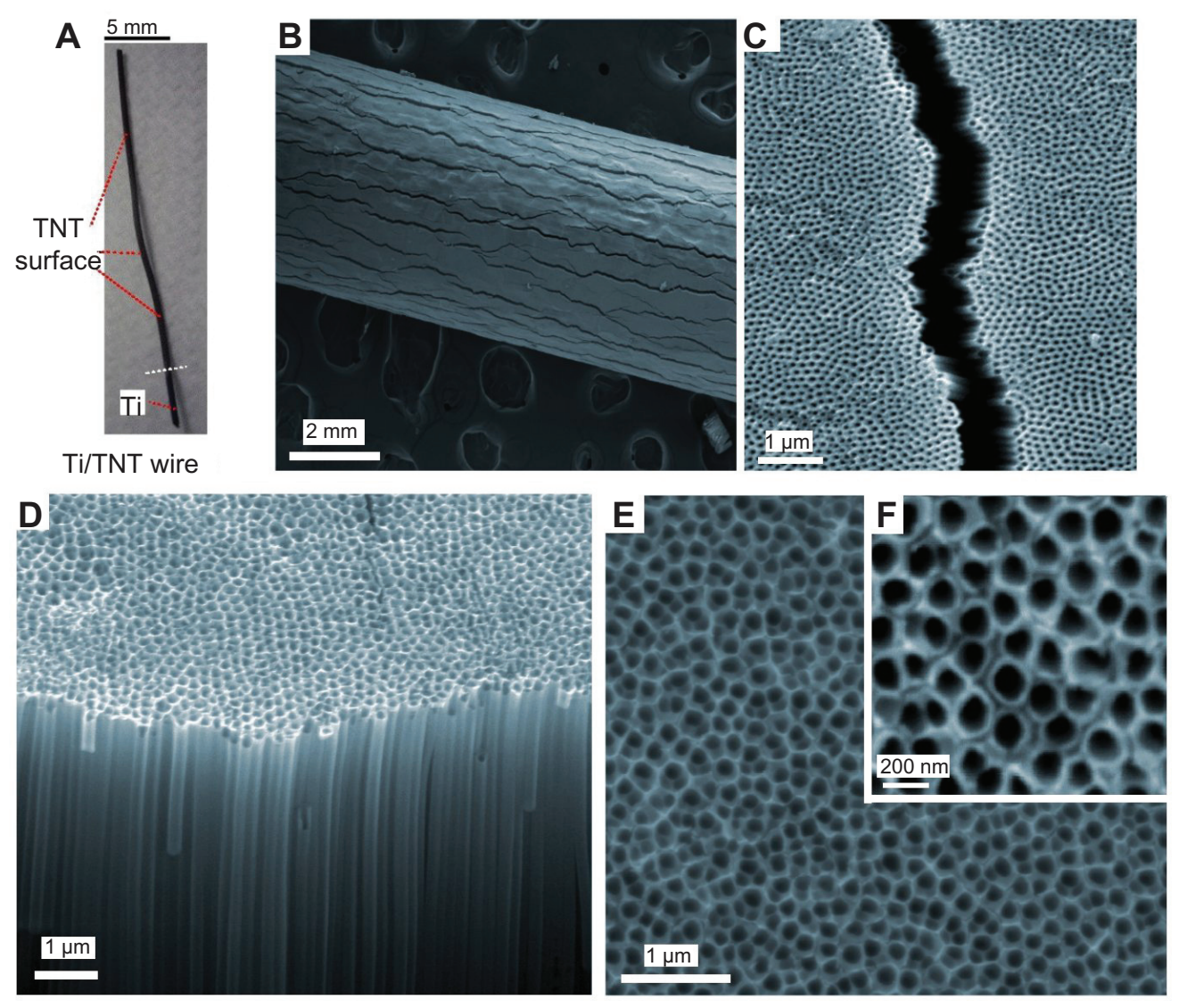

Figure 2 SEM images of titania nanotube (TNT) arrays generated on Ti wires by electrochemical anodization. (A) TNT/Ti wire implant, (B and C) low and high resolution image showing fractures of TNT film, (D-F) cross-sectional image and the top TNT surface showing typical nanotube structures.

Abbreviations: SEM, scanning electron microscope; TNT, titania nanotube; Ti, titanium. 
curved Ti wire. The SEM images show randomly dispersed vertical cracks (width $1-1.5 \mu \mathrm{m}$ and $>1 \mathrm{~mm}$ long) across the entire wire length (Figure $2 \mathrm{~B}$ and $\mathrm{C}$ ). The formation of these cracks is explained by radial growth of TNT film and mechanical stress caused by volume extension of the formed TNT oxide layer on the circular surface of the Ti wire. These cracks were not observed on TNT films grown on planar Ti surfaces, but their presence on wires did not significantly change the mechanical stability and rigidity of the TNT films. A high-resolution SEM images of the top surface and cross-sections of the TNT layer show a vertically aligned and densely packed array of uniform nanotubes with diameter of $170 \pm 10 \mathrm{~nm}$ and length $70 \pm 2 \mu \mathrm{m}$, across the entire structure (Figure 2D and E). These structural characteristics suggest that the TNT platforms are ideal for development of drug-releasing implants for local drug delivery applications. In particular, their wire form and their size $(0.75 \mathrm{~mm}$ dia $\times 10-20 \mathrm{~mm}$ length) offers considerable advantages for brain implants in comparison with commercial large polymer discs (14.5 mm diameter, Gliadel R Wafer, MGI Pharma). TNT/Ti wires can enter narrow gaps and occupy minimal space without obstruction of the brain tissues, potentially allowing simpler surgical procedures during implantation and the removal of implants.

\section{Drug loading characteristics of TNT/Ti wires}

TGA of the TNT/Ti wires was performed to confirm that drugs were successfully loaded into the nanotube structures and to determine the amount of loading. Figure 3 shows thermograms of the total weight loss of the TNT/Ti wires loaded with DOPA and DOXO. Since Ti and titanium oxide are thermally stable up to $2500^{\circ} \mathrm{C}$ to $3000^{\circ} \mathrm{C}$ respectively, they do not undergo any changes in the furnace, so the observed weight loss is used to determine the loaded drug

A

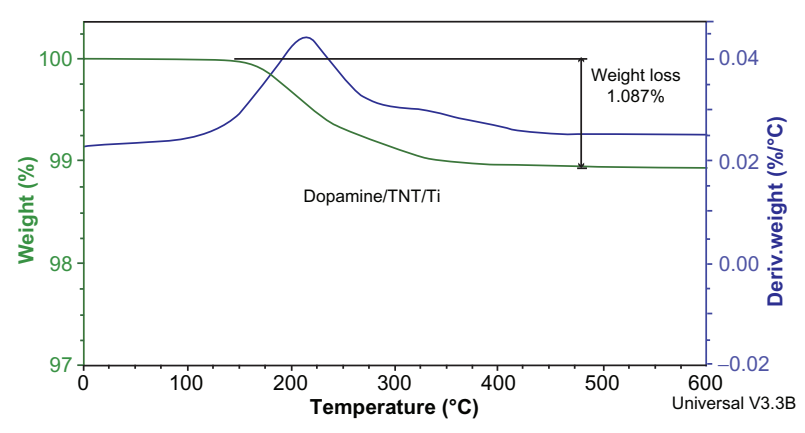

mass inside the TNTs. A one-step decrease in the thermogram was obtained which has the characteristic range matching the decomposition temperature of DOPA and DOXO. TGA results show total drug loadings of $170 \mu \mathrm{g}$ and $1200 \mu \mathrm{g}$ for DOPA and DOXO respectively in prepared TNT/Ti wire. These drugs have considerably different dosages recommended for systematic administration and these differences in their loading in TNTs were achieved using different loading conditions (drug concentration, loading time and postloading washing). Particularly, for the anticancer drug DOXO to be effective against cancer cells it is critical to have high concentrations $\left(>300 \mathrm{mg} / \mathrm{m}^{2}\right)$. In comparison with commercial brain implants in the form of polymer disc (Gliadal R, MGI Pharma), about 4-5 implants with TNT/Ti wires (length $15 \mathrm{~mm}$ ) are required to have the same drug loading capacity (>7 mg). However, the drug loading capacity of TNT/Ti wires can further be optimized by fabrication processes to increase nanotube dimensions (selecting appropriate electrolyte, voltage and time), by surface modifications, and by selecting the appropriate size/shape of the implant (wires, needles, plates or discs). Hence it is possible to customize TNT/Ti platforms to meet specific requirements for implantable drug delivery in the brain depending on the required dosage, properties of drugs, surgery requirements, and the the time of proposed brain therapy. ${ }^{18,19,31,32}$

\section{In vitro drug release of DOPA and DOXO from TNT/Ti wires}

Drug release profiles and characteristics of DOPA and DOXO loaded into TNT/Ti wire are presented in Figure 4 and Table 1. The drug release kinetics can be described in two phases, showing an initial burst release during the first 6 hours, followed by the second stage with slow release of the remaining drug. In the burst release phase, about $60 \%(100 \mu \mathrm{g})$ of DOPA and $25 \%(300 \mu \mathrm{g})$

B

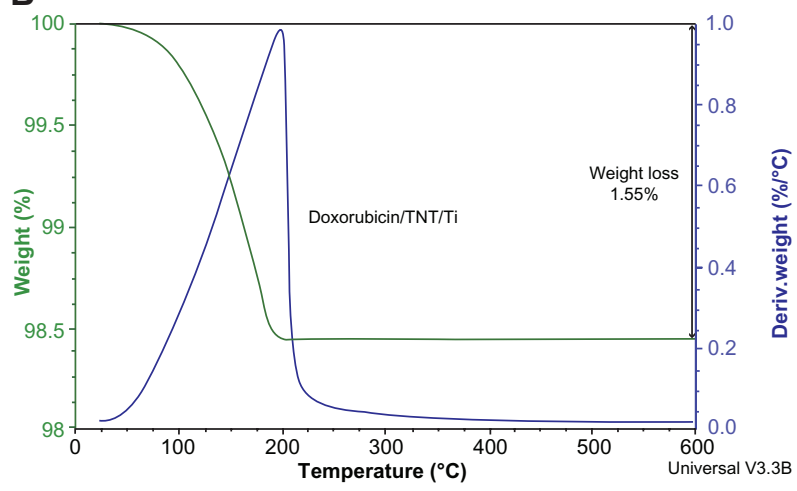

Figure 3 Thermogravimetric (TGA) graphs showing drug loading of (A) dopamine and (B) doxorubicin into TNT/Ti wire. Abbreviations: TGA, thermogravimetric analysis; TNT, titania nanotube; $\mathrm{Ti}$, titanium. 
A

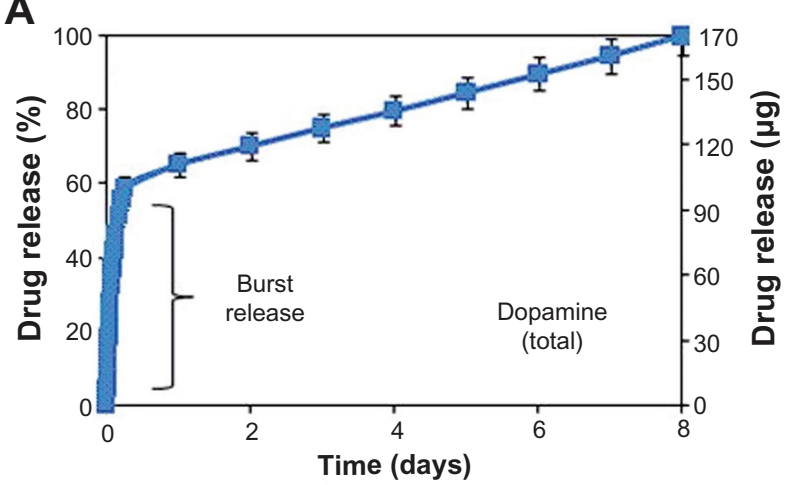

C

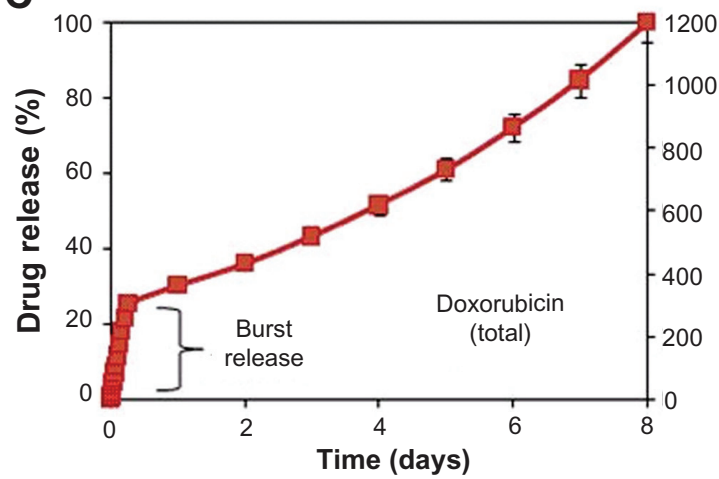

B

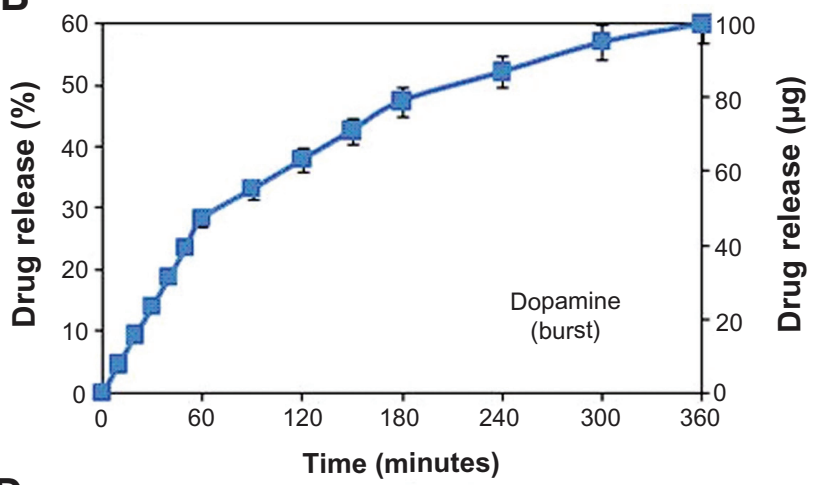

D

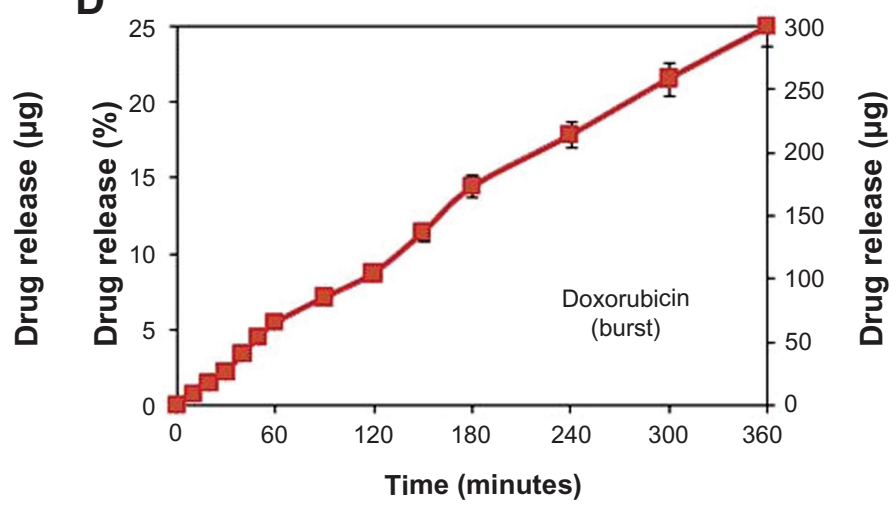

Figure 4 Drug release graphs showing cumulative release (\% and mass) from TNTs/Ti implants for (A and $\mathbf{B})$ dopamine and (C and $\mathbf{D})$ doxorubicin. Both total and burst releases are presented.

Abbreviations: TNT, titania nanotube; Ti, titanium.

of DOXO was released (Figure 4B and D). The fast initial release accounts for the fast diffusion of the drug molecules physisorbed on the top and upper channels of the TNTs, as a result of the high concentration gradient at the TNT interface with the bulk phosphate buffer solution. The difference in the release rate of the two drugs can be explained by differences in their solubility in buffer solution, which influences their diffusion rate into the bulk solution. Watersolubilities of DOXO and DOPA are $10 \mathrm{mg} / \mathrm{mL}$ and $600 \mathrm{mg} /$ $\mathrm{mL}$ respectively. Also the smaller size and molecular weight of DOPA (molecular weight, MW $=189.64 \mathrm{~g} / \mathrm{moL}$ ) as compared to DOXO $(\mathrm{MW}=543.5 \mathrm{~g} / \mathrm{mol})$ resulted in its faster uptake from the TNTs into the buffer solution.
In the second phase, different drug release kinetics were observed from TNT/Ti, with very slow and linearly increasing cumulative release over a period of 8 days (Figure 4A and C). The release mechanism of this phase is diffusion-controlled and it applies to the whole nanotube structure, reaching down its entire depth to $70 \mu \mathrm{m}$. Again slight differences between DOPA and DOXO were observed. Considering the high surface area and long capillary-like tubular structures of TNT, the diffusion of drug molecules to the buffer solution can be described as a surface-dependent phenomenon. The best-fitting model to the DOXO release data was observed using Higuchi and zero-order release, which describe drug release from an insoluble matrix. ${ }^{33}$

Table I Drug release characteristics of two drugs (dopamine and doxorubicin) from TNT/Ti wire showing their drug loading and release characteristics (\% and mass) during burst and 8-days release period

\begin{tabular}{|c|c|c|c|c|c|c|c|}
\hline \multirow{2}{*}{$\begin{array}{l}\text { Drug loaded into } \\
\text { TNT/Ti }\end{array}$} & \multirow{2}{*}{$\begin{array}{l}\text { Amount } \\
\text { loaded } \\
\text { drug }\end{array}$} & \multicolumn{4}{|c|}{ Cumulative drug release $(\%$ and $\mu \mathrm{g})$} & \multicolumn{2}{|c|}{ Rate of drug release } \\
\hline & & 6 hours & 2 days & 4 days & 8 days & Burst release & $\begin{array}{l}\text { After burst } \\
\text { release }\end{array}$ \\
\hline \multirow[t]{2}{*}{ Dopamine } & $170 \mu \mathrm{g}$ & $60 \%$ & $70 \%$ & $80 \%$ & $100 \%$ & $17 \mu \mathrm{g} / \mathrm{hour}$ & $9 \mu \mathrm{g} /$ day \\
\hline & & $100 \mu \mathrm{g}$ & $120 \mu g$ & $135 \mu \mathrm{g}$ & $170 \mu \mathrm{g}$ & & \\
\hline \multirow[t]{2}{*}{ Doxorubicin } & $1200 \mu \mathrm{g}$ & $25 \%$ & $36 \%$ & $50 \%$ & $100 \%$ & $50 \mu g /$ hour & $\mathrm{I} / 2 \mu \mathrm{g} /$ day \\
\hline & & $300 \mu \mathrm{g}$ & $435 \mu \mathrm{g}$ & $620 \mu g$ & $1200 \mu \mathrm{g}$ & & \\
\hline
\end{tabular}

Abbreviations: TNT, titania nanotube; Ti, titanium. 
The square root of a time-dependent process is based on Fickian diffusion law where the diffusion-controlled release rate of drug molecules decreases as a function of time due to a reduction in the concentration gradient. ${ }^{33}$ This behavior can be explained by the influence of several parameters including low drug solubility, larger molecular size, and chemistry. DOXO molecules have a higher number of $\mathrm{OH}$ groups and one positively charged amino group, which could interact with the TNT surface in different ways, including hydrogen bonding and electrostatic interactions, and causing their slower release from the nanotube structures. Pharmaceutical dosage following a zero-ordered pattern is the ideal profile for drug release, because it provides the same amount of drug elution per unit of time. These results confirmed that TNT/Ti wire can be successfully employed as a suitable drug-releasing platform for local delivery of CNS transmitters such as DOPA and anticancer drugs such as DOXO, to combat brain neurodegenerative disorders and brain tumors with acceptable dosage and predictable release kinetics. ${ }^{18,32,34,35}$

Our results demonstrated that the drug release into the local environment during this time was constant, with a value of about $9 \mu \mathrm{g}$ and $112 \mu \mathrm{g}$ per day for DOPA and DOXO respectively. By controlling the dimensions of TNT structures (diameter and length), this local concentration can be controlled and tuned to fit the optimal therapeutic window for the treatment of brain cancer or neurodegenerative diseases. ${ }^{4,34,35}$ The general approach for brain cancer treatments using implantable devices requires a large drug loading and constant release over extended periods (5-6 weeks). To address this problem, we recently introduced several approaches to considerably extend drug release from TNTs prepared on planar surfaces using polymer micelles and polymer coatings (plasma polymers, chitosan, poly[lactic-coglycolic acid]). ${ }^{24-28}$ These approaches can be incorporated on the wire implants to achieve a long and sustained release of specific therapeutics with desired concentration and zeroorder kinetics for more than 4-6 weeks.

\section{Conclusion}

This study presents the fabrication of titanium wires with titania nanotube arrays and explores their drug loading and release characteristics for two drugs, DOPA: a neurotransmitter agent and DOXO: an anticancer drug, both important for local and implantable drug delivery in the brain. TNTs on Ti wire were fabricated by a simple and scalable nanofabrication technology based on an electrochemical anodization process, with ability to tune dimensions to load substantial amounts of brain therapeutic drugs ( $>1 \mathrm{mg}$ per implant).
It is proposed that these tiny and biocompatible wire implants can easily be inserted in the brain using microsurgery and provide considerable advantages in comparison with existing clinically proven brain implants. These advantages include: appropriate shape (needles and wires), which requires less invasive surgery; loading/release of water-soluble and insoluble drugs or drug-carriers; tailorable drug release characteristics including sustained and stimulated release; and a wide applications for different brain therapies (eg, neurodisorders and cancer). Future investigations are necessary to prove the in vivo performance of these implants.

\section{Acknowledgments}

The authors acknowledge the financial support of the Australian Research Council (DP 0770930 and DP 120101680) for this work. Professor Andreas Evdokiou of the Basil Hetzel Institute, Queen Elizabeth Hospital and Adelaide University is especially acknowledged for supply of the DOXO drug.

\section{Disclosure}

The authors report no conflict of interest in this work.

\section{References}

1. Mainardes RM, Silva LP. Drug delivery systems: past, present, and future. Curr Drug Targets. 2004;5(5):449-455.

2. LaVan DA, McGuire T, Langer R. Small-scale systems for in vivo drug delivery. Nat Biotechnol. 2003;21(10):1184-1191.

3. Pardridge WM. Blood-brain barrier drug targeting: the future of brain drug development. Mol Interv. 2003;3(2):90-105, 51.

4. Wang PP, Frazier J, Brem H. Local drug delivery to the brain. Adv Drug Deliv Rev. 2002;54(7):987-1013.

5. Groothuis DR. The blood-brain and blood-tumor barriers: a review of strategies for increasing drug delivery. Neuro Oncol. 2000;2(1): 45-59.

6. Baratchi S, Kanwar RK, Khoshmanesh K, et al. Promises of nanotechnology for drug delivery to brain in neurodegenerative diseases. Curr Nanosci. 2009;5(1):15-25.

7. Bodor N, Buchwald P. Recent advances in the brain targeting of neuropharmaceuticals by chemical delivery systems. Adv Drug Deliv Rev. 1999;36(2-3):229-254.

8. Lesniak MS, Brem H. Targeted therapy for brain tumours. Nat Rev Drug Discov. 2004;3(6):499-508.

9. Au JL, Jang SH, Wientjes MG. Clinical aspects of drug delivery to tumors. J Control Release. 2002;78(1-3):81-95.

10. Laquintana V, Trapani A, Denora N, Wang F, Gallo JM, Trapani G. New strategies to deliver anticancer drugs to brain tumors. Expert Opin Drug Deliv. 2009;6(10):1017-1032.

11. Gilert A, Machluf M. Nano to micro delivery systems: targeting angiogenesis in brain tumors. J Angiogenes Res. 2010;2(1):20.

12. Raghavan R, Brady ML, Rodríguez-Ponce MI, Hartlep A, Pedain C, Sampson JH. Convection-enhanced delivery of therapeutics for brain disease, and its optimization. Neurosurg Focus. 2006;20(4):E12.

13. Oh S, Odland R, Wilson SR, et al. Improved distribution of small molecules and viral vectors in the murine brain using a hollow fiber catheter. J Neurosurg. 2007;107(3):568-577.

14. Scott AW, Tyler BM, Masi BC, et al. Intracranial microcapsule drug delivery device for the treatment of an experimental gliosarcoma model. Biomaterials. Apr 2011;32(10):2532-2539. 
15. Morrison PF, Chen MY, Chadwick RS, Lonser RR, Oldfield EH. Focal delivery during direct infusion to brain: role of flow rate, catheter diameter, and tissue mechanics. Am J Physiol. 1999;277(4 Pt 2):R1218-R1229.

16. Tyler B, Fowers KD, Li KW, et al. A thermal gel depot for local delivery of paclitaxel to treat experimental brain tumors in rats. J Neurosurg. 2010;113(2):210-217.

17. Brem H, Mahaley MS Jr, Vick NA, et al. Interstitial chemotherapy with drug polymer implants for the treatment of recurrent gliomas. J Neurosurg. 1991;74(3):441-446.

18. Weinberg BD, Blanco E, Gao J. Polymer implants for intratumoral drug delivery and cancer therapy. J Pharm Sci. 2008;97(5):1681-1702.

19. Lin SH, Kleinberg LR. Carmustine wafers: localized delivery of chemotherapeutic agents in CNS malignancies. Expert Rev Anticancer Ther. 2008;8(3):343-359.

20. Ghicov A, Schmuki P. Self-ordering electrochemistry: a review on growth and functionality of $\mathrm{TiO}_{2}$ nanotubes and other self-aligned MO(x) structures. Chem Commun (Camb). 2009;28(20):2791-2808.

21. Popat KC, Eltgroth M, LaTempa TJ, Grimes CA, Desai TA. Titania nanotubes: a novel platform for drug-eluting coatings for medical implants. Small. 2007;3(11):1878-1881.

22. Losic D, Simovic S. Self-ordered nanopore and nanotube platforms for drug delivery applications. Expert Opin Drug Deliv. 2009;6(12):1363-1381.

23. Smith LJ, Swaim JS, Yao C, Haberstroh KM, Nauman EA, Webster TJ. Increased osteoblast cell density on nanostructured PLGA-coated nanostructured titanium for orthopedic applications. Int J Nanomedicine. 2007;2(3):493-499.

24. Aw MS, Simovic S, Addai-Mensah J, Losic D. Polymeric micelles in porous and nanotubular implants as a new system for extended delivery of poorly soluble drugs. J Mater Chem. 2011;21(20):7082-7089.

25. Aw MS, Gulati K, Losic D. Controlling drug release from titania nanotube arrays using polymer nanocarriers and biopolymer coating. J Biomater Nanobiotechnol. 2011;2:477-484.
26. Simovic S, Losic D, Vasilev K. Controlled drug release from porous materials by plasma polymer deposition. Chem Commun (Camb). 2010;46(8):1317-1319.

27. Gulati K, Ramakrishnan S, Aw MS, Atkins GJ, Findlay DM, Losic D. Biocompatible polymer coating of titania nanotube arrays for improved drug elution and osteoblast adhesion. Acta Biomater. 2012;8(1): 449-456.

28. Gulati K, Aw MS, Losic D. Drug-eluting Ti wires with titania nanotube arrays for bone fixation and reduced bone infection. Nanoscale Res Lett. 2011;6:571-577.

29. Vasilev K, Poh Z, Kant K, Chan J, Michelmore A, Losic D. Tailoring the surface functionalities of titania nanotube arrays. Biomater. 2010;31(3):532-540.

30. Krishna K, Losic D. A simple approach for synthesis of TiO nanotubes with through-hole morphology. Physica Status Solidi RRL. 2009;3(5): 139-141.

31. Aw MS, Addai-Mensah J, Losic D. Multi-drug delivery system with sequential release using titania nanotube arrays. Chem Commun (Camb). 2012;48(27):3348-3350.

32. Aw MS, Addai-Mensah J, Losic D. Magnetic-responsive delivery of drug carriers using titania nanotube arrays. J Mater Chem. 2012; 22(14):6561-6563.

33. Siepmann J, Lecomte F, Bodmeier R. Diffusion-controlled drug delivery systems: calculation of the required composition to achieve desired release profiles. J Control Release. 1999;60(2-3):379-389.

34. López T, Bata-García JL, Esquivel D, et al. Treatment of Parkinson's disease: nanostructured sol-gel silica-dopamine reservoirs for controlled drug release in the central nervous system. Int J Nanomedicine. 2010;6: 19-31.

35. D'Aurizio E, Sozio P, Cerasa LS, et al. Biodegradable microspheres loaded with an anti-Parkinson prodrug: an in vivo pharmacokinetic study. Mol Pharm. 2011;8(6):2408-2415.
International Journal of Nanomedicine

\section{Publish your work in this journal}

The International Journal of Nanomedicine is an international, peerreviewed journal focusing on the application of nanotechnology in diagnostics, therapeutics, and drug delivery systems throughout the biomedical field. This journal is indexed on PubMed Central, MedLine, CAS, SciSearch ${ }^{\circledR}$, Current Contents ${ }^{\circledR} /$ Clinical Medicine,

\section{Dovepress}

Journal Citation Reports/Science Edition, EMBase, Scopus and the Elsevier Bibliographic databases. The manuscript management system is completely online and includes a very quick and fair peer-review system, which is all easy to use. Visit http://www.dovepress.com/ testimonials.php to read real quotes from published authors. 